\title{
Randomisierte, kontrollierte Studie zur palliativen Radiotherapie von Kopf-Hals-Tumoren - Herausforderungen bleiben bestehen
}

\author{
Alexander Fabian ${ }^{1}$. David Krug ${ }^{1}$ - Jürgen Dunst ${ }^{1}$ \\ Online publiziert: 21. August 2020 \\ (c) Der/die Autor(en) 2020
}

Hintergrund In der palliativen Radiotherapie von KopfHals-Tumoren findet eine Vielzahl unterschiedlicher Regime Verwendung. Das optimale Regime ist unklar. Aufgrund der schlechten Prognose müssen Therapiedauer, biologisch effektive Dosis und potenzielle Akut- und Spättoxizität gut gegeneinander abgewogen werden. Eine niederländische randomisierte Studie von Al-Mamgani und Kollegen verglich in diesem Kontext zwei Radiotherapieregime.

Patienten und Methodik Es handelt sich um eine zweiarmige Phase-III-Studie an 6 niederländischen Zentren. Einschlusskriterien waren: lokal fortgeschrittenes oder fernmetastasiertes Plattenepithelkarzinom des Oropharynx, Hypopharynx oder Larynx, ein guter Allgemeinzustand (ECOG 0-2) und keine Indikation für eine kurativ intendierte Lokaltherapie. Ein relevantes Ausschlusskriterium war eine antineoplastische Vorbehandlung des Kopf-HalsTumors. Die Patienten wurden 1:1 randomisiert. „Arm 1“ bestand aus einem Kurzzeitregime mit 6 Fraktionen von 6 Gy zweimal wöchentlich bis 36 Gy Gesamtdosis. „Arm 2“ war ein Langzeitregime mit 16 Fraktionen von 3,125 Gy viermal wöchentlich bis $50 \mathrm{~Gy}$ Gesamtdosis. In beiden Armen erfolgte eine intensitätsmodulierte oder volumetrisch modulierte (Rotations-)Bestrahlung des makroskopischen Tumorvolumens, ggf. einschließlich von Lymphknotenmetastasen, mit einem CTV-Saum von $10 \mathrm{~mm}$ und einem PTVSaum von 3 bis $5 \mathrm{~mm}$ ohne Behandlung elektiver Volumina. Primärer Endpunkt war die Zeit bis zur lokoregionären

Originalpublikation Al-Mamgani A, Kessels R, Verhoef CG et al (2020) Randomized controlled trial to identify the optimal radiotherapy scheme for palliative treatment of incurable head and neck squamous cell carcinoma. Radiotherapy and Oncology, https://doi.org/10.1016/j.radonc.

\footnotetext{
Dr. med. Alexander Fabian alexander.fabian@uksh.de

1 Klinik für Strahlentherapie, Universitätsklinikum Schleswig-Holstein (UKSH), Campus Kiel, Arnold-Heller-Str. 3, 24105 Kiel, Deutschland
}

Progression. Die Autoren postulierten eine Verlängerung der Zeit bis zur lokoregionären Progression um mindestens 10 Wochen zugunsten von „Arm 2“. Sekundäre Endpunkte waren unter anderem das progressionsfreie Überleben, das Gesamtüberleben, die Toxizitätslast sowie die gesundheitsbezogene Lebensqualität. Die Fallzahlkalkulation mündete in 300 geplanten Patienten.

Ergebnisse In knapp 2 Jahren wurden 34 Patienten rekrutiert und randomisiert. Die Daten von 18 Patienten konnten in „Arm 1“ und von 14 Patienten in „Arm 2“ analysiert werden. Die Studie wurde aufgrund geringer Rekrutierung frühzeitig geschlossen. Alle Statistiken sind daher deskriptiv bzw. hypothesengenerierend. Die lokoregionäre Kontrolle betrug nach einem Jahr $57 \%$ in „Arm 1“ und $69 \%$ in ,Arm 2“ $(p=0,45)$. Die mediane Zeit bis zur lokoregionären Progression war in beiden Armen noch nicht erreicht. Während das mediane progressionsfreie Überleben 5 Monate beziehungsweise 8 Monate betrug, lag das mediane Gesamtüberleben in „Arm 1 “ bei knapp 9 Monaten und in ,Arm 2“ bei knapp 15 Monaten $(p=0,2)$. Patienten in „Arm 1“ hatten mit $17 \%$ gegenüber $57 \%$ im ,Arm 2“ signifikant weniger Grad-3-Toxizität, maßgeblich bedingt durch eine geringer ausgeprägte Mukositis. Die Mukositis führte bei 4 Patienten im ,Arm 2“ zu einem frühzeitigen Therapieabbruch. In „Arm 1“ brach lediglich ein Patient die Therapie frühzeitig ab. Die gesundheitsbezogene Lebensqualität unterschied sich in beiden Armen nicht maßgeblich. Beide Arme zeigten einen Trend zu einer gebesserten allgemeinen gesundheitsbezogenen Lebensqualität (EORTC QLQ-C30) sowie zu gebesserten Schluckbeschwerden (EORTC QLQH\&N35) und gebessertem bzw. stabilem Schmerzgeschehen (EQ-5D-5L VAS).

Schlussfolgerungen der Autoren Die vorzeitig abgebrochene Studie erlaubt keine zweifelsfreien Aussagen zur klinischen Überlegenheit eines der beiden Regime. Aufgrund der geringeren Toxizitätslast und statistisch nicht signifikant unterschiedlichen onkologischen Ergebnisse 
könnte das Kurzzeitregime $(6 \times 6 \mathrm{~Gy})$ jedoch gegenüber dem Langzeitregime $(16 \times 3,125 \mathrm{~Gy})$ favorisiert werden.

\section{Kommentar}

Patienten mit einem Kopf-Hals-Tumor ohne kurative Option überleben im Median weniger als ein Jahr [1]. Selbst eine palliative Erstlinientherapie mit Chemo- und Immuntherapie konnte dies trotz der strengen Patientenselektion nur auf 13 bzw. 15 Monate verbessern [2]. Zusätzlich schränken Symptome wie Schmerzen, Blutungen und Schluckbeschwerden die Lebensqualität stark ein [3]. Häufig erfolgt eine palliative Radiotherapie in diesem Kontext [4]. Das optimale Radiotherapieregime ist jedoch unklar. Letzteres liegt zum Teil an den heterogenen Patientenkollektiven, die sich bezüglich Allgemeinzustand, Symptomatik, Tumorausdehnung und Indikation für die palliative Therapieintention deutlich unterscheiden (primär metastasierter vs. lokal weit fortgeschrittener Tumor vs. Komorbiditäten) [5]. Dies erfordert eine individualisierte Therapieplanung. Bislang fehlten daher auch randomisierte, kontrollierte Studien, um zu einer allgemein verbindlichen Vorgehensweise zu kommen. Al-Mamgani und Kollegen sind in ihren Bemühungen zu beglückwünschen, diese Lücke zu schließen. Ihre randomisierte Phase-III-Studie verglich ein Kurzzeitregime $(6 \times 6 \mathrm{~Gy})$ mit einem Langzeitregime $(16 \times 3,125 \mathrm{~Gy})$ zur palliativen Radiotherapie von Kopf-Hals-Tumoren. Mehrere Aspekte lassen es lohnenswert erscheinen, diese Studie näher zu reflektieren.

Erstens wurde die Studie frühzeitig abgebrochen. Anstatt der insgesamt 300 avisierten Patienten rekrutierten 6 niederländische Studienzentren in knapp 2 Jahren lediglich $34 \mathrm{~Pa}$ tienten. Dies veranschaulicht deutlich, wie schwierig es ist, in dieser Therapiesituation randomisierte strahlentherapeutische Studien durchzuführen, und könnte ein Spiegel der großen Heterogenität bei begrenzter Fallzahl sein.

Zweitens übertrifft das erreichte mediane Gesamtüberleben von 9 bzw. 15 Monaten teils deutlich das Gesamtüberleben der meisten bisher publizierten Kollektive mit nur ca. 6 Monaten [6]. Der gute Allgemeinzustand der eingeschlossenen Patienten (75\% ECOG 0-1; 25\% ECOG 2) könnte dies begründen; er deckt sich allerdings nur zum Teil mit unseren eigenen alltäglichen klinischen Erfahrungen. Ein weiterer Gesichtspunkt: Eine simultane Systemtherapie zur Radiotherapie war im Protokoll zwar nicht vorgesehen; inwieweit jedoch eine anschließende Chemo- und/oder Immuntherapie eingesetzt wurde und das Überleben beeinflusst haben könnte, wird in der Publikation nicht berichtet.

Auch wenn die statistische Auswertung bei 34 Patienten rein deskriptiv ist, zeigte sich drittens zwar ein numerischer Vorteil im Langzeitregime bezüglich lokaler Kontrolle und Gesamtüberleben, dieser war jedoch statistisch nicht signi- fikant. Demgegenüber bestand eine signifikant höhere Toxizitätslast im Langzeitregime, die auch zu mehr Therapieabbrüchen führte. Sowohl die onkologischen Endpunkte als auch die Toxizitätslast stehen im Einklang mit einarmigen Studien, die entweder ein Kurzzeit- oder ein Langzeitregime untersuchten. Fortin und Kollegen untersuchten beispielsweise 5 Fraktionen von $5 \mathrm{~Gy}$ an aufeinander folgenden Werktagen bei intensitätsmodulierter Radiotherapietechnik [7]. Das mediane Gesamtüberleben von 33 Patienten lag bei 6,5 Monaten, während lediglich 7\% eine Radiomukositis über Grad 2 aufwiesen. In einer einarmigen Vorläuferstudie untersuchten Al-Mamgani und Kollegen das Langzeitregime mit 16 Fraktionen von 3,215 Gy, jedoch ohne intensitätsmodulierte Radiotherapietechnik [8]. Das mediane Überleben von 158 Patienten lag hier bei 17 Monaten. Eine akute Radiomukositis über Grad 2 lag bei $65 \%$ der Patienten vor. Der augenfällige Unterschied im medianen Überleben könnte unter anderem durch einen deutlich höheren Anteil an Patienten mit fernmetastasierten Erkrankungen in der Studie von Fortin und Kollegen bedingt sein (knapp $25 \%$ vs. $9 \%$ ).

Viertens waren ,patient-reported outcomes“ zur Erhebung der gesundheitsbezogenen Lebensqualität lediglich ein sekundärer Endpunkt. In der palliativen Situation mit begrenzter Lebenserwartung sollte aber die gesundheitsbezogene Lebensqualität im Vordergrund stehen [9]. Bislang gibt es jedoch kaum Studien in diesem Kontext, die die gesundheitsbezogene Lebensqualität durch eine validierte Erhebung als primären Endpunkt einsetzen. Letzteres wäre wichtig, um den Wert der palliativen Radiotherapie von Kopf-Hals-Tumoren zu sichern, der zum Teil bereits infrage gestellt worden ist [10]. Aus diesem Grund haben wir eine prospektive multizentrische Beobachtungsstudie mit dem primären Endpunkt der gesundheitsbezogenen Lebensqualität initiiert (DRKS00021197).

\section{Fazit}

Zusammenfassend liegen die Herausforderungen in der palliativen Radiotherapie von Kopf-Hals-Tumoren in dem heterogenen Patientenkollektiv und der individuell abzuwägenden Balance zwischen Toxizität und antineoplastischer Effektivität. Während Patienten in reduziertem Allgemeinzustand von einem Kurzzeitregime mit geringerer Toxizitätslast profitieren könnten, könnte fitteren Patienten eine langfristigere Tumorkontrolle durch ein Langzeitregime ermöglicht werden. Der Fokus sollte dabei insbesondere auf einer Stabilisierung oder Verbesserung der gesundheitsbezogenen Lebensqualität liegen, was durch weitere Studien belegt werden muss.

Alexander Fabian, David Krug und Jürgen Dunst, Kiel 
Funding Open Access funding provided by Projekt DEAL.

Interessenkonflikt A. Fabian und J. Dunst geben an, dass kein Interessenkonflikt besteht. D. Krug erhielt Honorare von Merck Sharp \& Dome.

Open Access Dieser Artikel wird unter der Creative Commons Namensnennung 4.0 International Lizenz veröffentlicht, welche die Nutzung, Vervielfältigung, Bearbeitung, Verbreitung und Wiedergabe in jeglichem Medium und Format erlaubt, sofern Sie den/die ursprünglichen Autor(en) und die Quelle ordnungsgemäß nennen, einen Link zur Creative Commons Lizenz beifügen und angeben, ob Änderungen vorgenommen wurden.

Die in diesem Artikel enthaltenen Bilder und sonstiges Drittmaterial unterliegen ebenfalls der genannten Creative Commons Lizenz, sofern sich aus der Abbildungslegende nichts anderes ergibt. Sofern das betreffende Material nicht unter der genannten Creative Commons Lizenz steht und die betreffende Handlung nicht nach gesetzlichen Vorschriften erlaubt ist, ist für die oben aufgeführten Weiterverwendungen des Materials die Einwilligung des jeweiligen Rechteinhabers einzuholen.

Weitere Details zur Lizenz entnehmen Sie bitte der Lizenzinformation auf http://creativecommons.org/licenses/by/4.0/deed.de.

\section{Literatur}

1. Ledeboer QCP, van der Schroeff MP, Pruyn JFA et al (2011) Survival of patients with palliative head and neck cancer. Head Neck 33:1021-1026. https://doi.org/10.1002/hed.21572

2. Burtness B, Harrington KJ, Greil R et al (2019) Pembrolizumab alone or with chemotherapy versus cetuximab with chemotherapy for recurrent or metastatic squamous cell carcinoma of the head and neck (KEYNOTE-048): a randomised, open-label, phase
3 study. Lancet 394:1915-1928. https://doi.org/10.1016/S01406736(19)32591-7

3. Lokker ME, Offerman MPJ, van der Velden L-A et al (2013) Symptoms of patients with incurable head and neck cancer: prevalence and impact on daily functioning. Head Neck 35:868-876. https:// doi.org/10.1002/hed.23053

4. Mayland CR, Ingarfield K, Rogers SN et al (2020) Disease trajectories, place and mode of death in people with head and neck cancer: findings from the "head and neck 5000" population-based prospective clinical cohort study. Palliat Med. https://doi.org/10.1177/ 0269216320904313

5. Grewal AS, Jones J, Lin A (2019) Palliative radiation therapy for head and neck cancers. Int J Radiat Oncol Biol Phys 105:254-266. https://doi.org/10.1016/j.ijrobp.2019.05.024

6. Shahid Iqbal M, Kelly C, Kovarik J et al (2018) Palliative radiotherapy for locally advanced non-metastatic head and neck cancer: a systematic review. Radiother Oncol 126:558-567. https://doi.org/ 10.1016/j.radonc.2017.12.011

7. Fortin B, Khaouam N, Filion E et al (2016) Palliative radiation therapy for advanced head and neck carcinomas: a phase 2 study. Int J Radiat Oncol Biol Phys 95:647-653. https://doi.org/10.1016/ j.ijrobp.2016.01.039

8. Al-mamgani A, Tans L, Van rooij PHE et al (2009) Hypofractionated radiotherapy denoted as the "Christie scheme": an effective means of palliating patients with head and neck cancers not suitable for curative treatment. Acta Oncol 48:562-570. https://doi.org/ 10.1080/02841860902740899

9. Kelley AS, Morrison RS (2015) Palliative care for the seriously ill. N Engl J Med 373:747-755. https://doi.org/10.1056/NEJMra 1404684

10. Nilsen ML, Johnson JT (2017) Potential for low-value palliative care of patients with recurrent head and neck cancer. Lancet Oncol 18:e284-e289. https://doi.org/10.1016/S1470-2045(17)30260-7 